\title{
Providing optimal interventional oncology procedures at one of the COVID-19 referral center in Italy
}

\author{
Filippo Pesapane ${ }^{1} \cdot$ Anna Maria lerardi $^{2} \cdot$ Antonio Arrichiello $^{2} \cdot$ Gianpaolo Carrafiello $^{1,2}$ (])
}

Received: 1 May 2020 / Accepted: 29 July 2020 / Published online: 9 August 2020

๑) Springer Science+Business Media, LLC, part of Springer Nature 2020

\begin{abstract}
The COVID-19 pandemic has deeply impacted the activity of interventional oncology in cancer centers. Since the first COVID case was diagnosed in Italy on February 21 st, our Hospital, located in Milan downtown, has been at the frontline to manage this emergency and to try to ensure essential services. In the present article, we discuss the changes that need to be done for the organization, safety, and patient management in interventional oncology.
\end{abstract}

Keywords Interventional oncology · COVID-19 · Italy

Since the novel coronavirus disease (COVID-19) was first identified in Wuhan (Huebei Province, China) in December 2019 [1], it has spread globally resulting in the ongoing 2019-20 coronavirus pandemic [2] with almost two million confirmed cases, and more than two-hundred countries involved, on April 16th, based on the WHO situation report [3].

From the second half of February 2020, a rapid and unexpected surge of patients with COVID-19 is posing a serious threat to the Italian National Health Service, especially in Lombardy that, with 62,153 confirmed COVID-19 patients and 11,377 deaths, on Apri1 6th, it is the most hard-hit province in all of Italy during the pandemic [4].

Our hospital is located in Milan, capital of the Lombardy, and currently it serves as one of the national referral centers for patients with severe COVID-19. To optimize the available healthcare resources, the Lombardy government made a differentiation between elective and non-deferrable procedures from the early period, defining which patients could be treated, namely only emergency and oncologic patients [5]. Accordingly, most of the interventional oncology procedures are continuing as normal in our center even under such extreme circumstances. The goals are to continue to

Anna Maria Ierardi

amierardi@yahoo.it

1 Department of Health Sciences, Università Degli Studi di Milano, Milan, Italy

2 Radiology Department, Fondazione IRCCS Cà Granda, Ospedale Maggiore Policlinico, Milan, Italy provide optimal care while reducing infective risk to patients and to staff. Using the appropriate personal protective equipment (PPE) and innovative operating protocols specifically designed for the pandemic [6], we continue to deploy vascular accesses for chemiotherapy, to perform imaging-guided biopsies, tumor ablations, transarterial radioembolization and chemoembolization therapies. Moreover, we continue to provide palliative procedures such as tunneled peritoneal and pleural catheters, drainage for fluid collections and biliary drainage in pancreas cancer and cholangiocarcinoma, and we also performed every kind of embolization whenever deemed to be necessary on a daily basis.

As cancer patients are a vulnerable population, especially during COVID-19 outbreak, the risks, benefits, and comorbidities of each patient's case must be carefully considered individually.

Although the IR workflow has been reorganized to limit the risk of transmission between patients, and between patients and healthcare workers, we have made no significant changes in our procedure workflows to keep outpatients and inpatients in the same area. However, all patients undergo to reverse transcription polymerase chain reaction (rRTPCR) test performed on respiratory samples obtained by a nasopharyngeal swab the day before to avoid the risk of cross-contamination. Moreover, we are not keeping patients overnight for observation unless medically necessary.

All staff underwent training for proper donning/doffing of PPE, and we have done rehearsals as well as the real thing. Notably, in our department no incidents between noninfected and infected patients have been documented so far, 
and there has been no evidence of COVID-19 infection of healthcare workers in the IR service to our knowledge. Additionally, we recently obtained a backup call team to serve as the "clean team" for grabbing supplies and taking over after a procedure on a COVID positive/under investigation patient. Finally, we asked for clearance to test all asymptomatic inpatients but unfortunately our healthcare system cannot currently afford such number of testing bandwidth.

Funding This study was not supported by any funding.

\section{Compliance with ethical standards}

Conflict of interest The authors declare that they have no conflict of interest.

Ethical approval All procedures performed in studies involving human participants were in accordance with the ethical standards of the institutional and/or national research committee and with the 1964 Helsinki Declaration and its later amendments or comparable ethical standards.

\section{References}

1. Coronavirus disease 2019 (COVID-19)-Symptoms and causes. Mayo Clinic. Retrieved 14 April 2020.

2. Hui DS, Azhar E, Madani TA, Ntoumi F, Kock R, Dar O, et al. The continuing 2019-nCoV epidemic threat of novel coronaviruses to global health - the latest 2019 novel coronavirus outbreak in Wuhan China. Int J Infect Dis. 2020;91(264):266. https://doi. org/10.1016/j.ijid.2020.01.009.

3. https://www.who.int/emergencies/diseases/novel-coronaviru s-2019/situation-reports/.

4. https://www.epicentro.iss.it/coronavirus/sars-cov-2-sorveglian za-dati.

5. Deliberazione ${ }^{\circ} \mathrm{XI} / 2906$, Seduta 8 March 2020.

6. Ierardi AM, Wood BJ, Gaudino C, Angileri SA, Jones EC, Hausegger K, Carrafiello G. How to handle a COVID-19 patient in the angiographic suite. Cardiovasc Intervent Radiol. 2020. https ://doi.org/10.1007/s00270-020-02476-8.

Publisher's Note Springer Nature remains neutral with regard to jurisdictional claims in published maps and institutional affiliations. 